\title{
SOBRECARGA E QUALIDADE DE VIDA DOS CUIDADORES INFORMAIS ASSISTIDOS POR UM SERVIÇO DE ATENÇÃO DOMICILIAR DO OESTE DO PARANÁ
}

\author{
OVERLOAD AND QUALITY OF LIFE OF INFORMAL CAREGIVERS \\ ASSISTED BY A HOME CARE SERVICE IN THE WEST OF PARANÁ
}

\author{
Sara Naomi Shimabukuro ${ }^{1 *}$, Juliana Emi Shimabukuro , Luciana Osorio Cavalli² \\ ${ }^{1}$ Acadêmica do curso de Medicina do Centro Universitário FAG. ${ }^{2}$ Graduada em Medicina, Mestre em \\ Biociências e Saúde, Docente do curso de Medicina do Centro Universitário FAG. \\ *Autor Correspondente: snshimabukuro@minha.fag.edu.br. https://orcid.org/0000-0002-5655-0584
}

DOI: $10.35984 / f j h . v 2 i 2.194$

\begin{abstract}
RESUMO
Contextualização: cuidador é a pessoa que auxilia o paciente em sua vida diária, sendo o cuidador informal aquele que exerce tal função sem formação profissional e capacitação. Objetivo: avaliar a sobrecarga e a qualidade de vida em cuidadores de pacientes assistidos por um serviço de atenção domiciliar de um município do Oeste do Paraná. Materiais e Métodos: os cuidadores informais submeteram-se à aplicação da Versão Brasileira da Escala Zarit Burden Interview (ZBI) e do Questionário de Avaliação da Sobrecarga do Cuidador Informal (QASCI). Resultados: verificou-se que $80 \%$ dos participantes da pesquisa sempre sentem que os pacientes assistidos dependem deles, $40 \%$ dos entrevistados se sentem moderadamente sobrecarregados e, em relação à piora da saúde dos mesmos pelo exercício da função, $20 \%$ relatam sempre apresentar esse sentimento e, $20 \%$, às vezes. O estudo propiciou também uma correlação entre os questionários aplicados, sendo que cada ponto adicional na Escala ZBI correspondeu a 0,8 ponto adicional no QASCI (IC 95\%). Conclusão: o estudo permite concluir que há sobrecarga moderada no grupo de cuidadores informais avaliado e conduz a uma reflexão acerca da necessidade do reconhecimento das demandas do cuidador informal.
\end{abstract}

Palavras-chave: Assistência domiciliar; Atenção à Saúde; Cuidadores; Qualidade de vida.

\begin{abstract}
Contextualization: caregiver is a person who assists the patient in its daily life and the informal caregiver is the one who performs this function without professional training or qualification. Objective: to evaluate caregivers' overload and their quality of life. These caregivers take care of patients assisted by a home care service from a west of Paraná municipality. Materials and Methods: informal caregivers underwent the application of the Brazilian Version of the Zarit Burden Interview Scale (ZBI) and to the Informal Caregiver Overload Assessment Questionnaire (QASCI). Results: it was found that $80 \%$ of the participants always feel that the patients assisted depend on them, $40 \%$ of the interviewees feel moderately overloaded, and about to the their health decline by the exercise of the function, $20 \%$ always report this feeling and, 20\%, sometimes. The study also provided a correlation between the questionnaires applied. So each additional point in the ZBI Scale corresponded to an additional 0.8 point in the QASCI $(95 \% \mathrm{Cl})$. Conclusion: the study concludes that there is moderate overload in the group of informal caregivers evaluated and it leads to a reflection about the need to recognize the demands of the informal caregiver.
\end{abstract}

Keywords: Home nursing; Health care; Caregivers; Quality of life. 


\section{INTRODUÇÃO}

O serviço de atenção domiciliar foi potencializado devido à necessidade de desospitalização para redução das taxas de infecção hospitalar e para proporcionar maior convivência familiar aos pacientes, de acordo com a Agência Nacional de Vigilância Sanitária. Este órgão regulamenta as empresas que prestam atenção domiciliar no Brasil aos pacientes clinicamente estáveis por meio de equipe multidisciplinar (ANVISA).

O Ministério da Saúde propôs as Modalidades de Atenção Domiciliar (AD). A Modalidade AD1 constitui-se na Atenção Básica aos pacientes que necessitam de cuidados de menor intensidade. O Melhor em Casa contempla, por sua vez, as Modalidades AD2 e AD3, que proporcionam aos pacientes uma maior frequência de cuidados e equipamentos específicos, respectivamente (Ministério da Saúde).

A Equipe Multiprofissional de Atenção Domiciliar (EMAD) visa, segundo a RDC/ANVISA no. 11 de 2006 à assistência clínico-terapêutica e psicossocial ao paciente em ambiente domiciliar (ANVISA, 2016). A composição da EMAD varia conforme a população do município e, de acordo com o Ministério da Saúde, a EMAD Tipo 1 assiste municípios com população de 40 mil habitantes ou mais enquanto a Tipo 2, entre 20 mil e 39.999 habitantes (Ministério da Saúde). O cuidador corresponde, por sua vez, à pessoa capacitada para auxiliar o paciente em suas necessidades e atividades de vida diária, sendo que os mesmos podem ou não apresentar vínculo familiar entre si (ANVISA, 2016). Os cuidadores apresentam certo grau de sobrecarga relacionada à execução de sua função (GUERRA et al, 2017).

O cuidador informal exerce função ainda mais complexa visto que realizam essa tarefa sem formação profissional. É necessário, portanto, a capacitação do cuidador informal para o seu bem-estar físico e psicológico, que apresentam repercussões positivas na qualidade do serviço prestado (CASTRO et al, 2017). A implementação de políticas públicas de suporte social é também importante aos cuidadores e familiares (DE SOUZA et al, 2015). O cuidador influencia, de maneira geral, na qualidade de vida do paciente ao executar suas funções de modo que a presente pesquisa visou a incrementação do conhecimento relacionado ao mesmo com ênfase na saúde do cuidador informal. O estudo teve como objetivo avaliar a sobrecarga e a qualidade de vida em cuidadores de pacientes assistidos por um serviço de atenção domiciliar de um município do Oeste do Paraná.

\section{METODOLOGIA}

Realizou-se um estudo quantitativo de natureza transversal e descritiva por meio de questionários aplicados aos cuidadores dos pacientes do Programa de Assistência e Internamento Domiciliar (Paid) em um município do Oeste do Paraná no período de setembro de 2017 à fevereiro de 2018. A aplicação dos questionários foi realizada com a privacidade dos cuidadores em horário agendado previamente com os ACS sob supervisão dos pesquisadores. O trabalho foi desenvolvido por meio da aplicação de dois questionários individuais compostos por 54 asserções ao todo. Ambos os questionários podem ser utilizados para avaliação periódica da sobrecarga física, emocional e social do cuidador (Caderno de Atenção Domiciliar Volume, 2012). Das 54 asserções, 22 eram provenientes da Versão Brasileira da Escala Zarit Burden Interview (ZBI), validada por Scazufca e regularizada para uso no Brasil. O resultado obtido pela Escala ZBI permite a graduação de sobrecarga dos cuidadores informais 
em: ausente ( 0 a 20 pontos), leve a moderado ( 21 a 40 pontos), moderado a severo (41 a 60 pontos) e intenso (61 a 88 pontos).

O restante das asserções correspondia ao Questionário de Avaliação da Sobrecarga do Cuidador Informal (QASCI), desenvolvido em Portugal, semanticamente adaptado e com as mesmas propriedades psicométricas para uso no Brasil. As respostas do QASCI foram referentes a frequência com que as situações ocorreram nas últimas 4 semanas anteriores ao dia da aplicação do questionário. Os 32 itens do QASCI integram sete dimensões, são elas: Implicações na vida pessoal (11 itens); Satisfação com o papel e com o familiar (cinco itens); Reações às exigências (cinco itens); Sobrecarga emocional (quatro itens); Apoio familiar (dois itens); Sobrecarga financeira (dois itens) e Percepção dos mecanismos de eficácia e de controle (três itens). O escore final resulta da soma total das respostas obtidas para os 32 itens referentes a cada domínio, divididos pelo número total de domínios ou por meio da média dos itens, após a inversão das pontuações dos itens das três dimensões positivas. Os valores mais altos são equivalentes a casos com maior peso ou maior sobrecarga. A pesquisa possibilitou avaliar a fidedignidade dos resultados obtidos e os domínios de maior sobrecarga, se realmente presente.

Os dados foram tabulados no software Microsoft Excel® 2013 para formulações e tabelas. A análise estatística dos dados foi realizada no software $R$ versão 3.5.1 ( $R$ Core Team, 2018) com confiabilidade de 95\%. Optou-se pela realização da correlação e da regressão linear simples entre as respostas dos questionários para avaliar a fidedignidade dos resultados obtidos e a existência de real sobrecarga dos participantes. O estudo respeitou as normas preconizadas pela Resolução 466/12 do Conselho Nacional de Saúde para a realização de pesquisas envolvendo seres humanos. O projeto foi aprovado pelo Comitê de Ética em Pesquisa com Seres Humanos da Faculdade Assis Gurgacz CEP-FAG sob o parecer de no 2231607, sendo que a pesquisa foi autorizada pela Secretaria Municipal de Saúde do município. Os participantes assinaram o Termo de Consentimento Livre e Esclarecido (TCLE) e o Termo de Compromisso para Uso de Dados em Arquivo, permitindo a aplicação dos instrumentos de pesquisa.

\section{RESULTADOS E DISCUSSÃO}

Os participantes da pesquisa foram recrutados pelos Agentes Comunitários de Saúde (ACS) por meio de agendamento. Dos cuidadores abordados, o total de 35 concordaram em participar da pesquisa e responder aos questionários. O perfil da população de estudo não foi caracterizado visto que o objetivo do mesmo estava relacionado à correlação dos questionários. As informações obtidas por meio da Escala ZBI e do QASCI estão detalhadas nas Tabelas 1 e 2. Os dados obtidos a partir da Escala ZBI demonstraram que $80 \%$ dos participantes da pesquisa sempre sentem que os pacientes assistidos dependem deles. Além disso, $40 \%$ dos entrevistados se sentem moderadamente sobrecarregados e, em relação à piora da saúde dos mesmos pelo exercício da função, $20 \%$ relatam sempre apresentar esse sentimento e, $20 \%$, às vezes.

A aplicação do QASCI permitiu inferir que 51,42\% dos entrevistados acreditam que executar tal função tem exigido grande esforço físico. Informou, ainda, que $54,28 \%$ dos participantes da pesquisa consideram ter conhecimentos e experiência para cuidar dos seus respectivos familiares. O estudo propiciou também uma correlação entre os questionários aplicados, sendo que cada ponto adicional na Escala ZBI correspondeu a 0,8 ponto adicional no QASCI (IC 95\%). Dessa forma, FAG Journal of Health - ISSN 2674-550X, 2020, v.2, n.2, p. 207

DOI: 10.35984/fjh.v2i2.194 
assim como pode ser visualizado no trabalho, quanto maior o resultado obtido em um, maior será no outro questionário de modo que a presença de sobrecarga foi equivalente.

Tabela 1. Distribuição da frequência do grau de sobrecarga dos cuidadores informais, segundo Escala ZBI. Cascavel-PR em 2017-2018.

\begin{tabular}{ccc}
\hline GRAU DE SOBRECARGA DOS & Frequência & $\%$ \\
CUIDADORES INFORMAIS* & & 14,28571 \\
Ausente & 5 & 48,57143 \\
Leve a Moderado & 17 & 34,28571 \\
Moderado a Severo & 12 & 2,85714 \\
Intenso & 1 & 100 \\
Total & 35 &
\end{tabular}

${ }^{*}$ Avaliado pela pontuação total da Escala ZBI.

Quando a pessoa se torna um cuidador informal, a sua vida é comumente reestruturada. Ocorrem desgastes físicos, emocionais e sociais de forma que a saúde e o bem-estar do mesmo são afetados (DO NASCIMENTO, 2015; FERNANDES e ANGELO, 2016), assim como redução do tempo para dedicar a si próprio (DE SOUZA et al, 2015), sendo que $51,42 \%$ dos entrevistados acreditam que executar tal função tem exigido grande esforço físico. O cuidador informal, por não ser profissionalmente preparado para prestar cuidados como o formal, enfrenta dificuldades em sua rotina de auxílio. Entretanto, é imprescindível que ele cuide de si, tanto para a o seu bemestar e quanto o do paciente (DE SOUZA et al, 2015).

Tabela 2. Escore total e dos domínios do QASCI para amostra estudada. Cascavel-PR em 20172018.

\begin{tabular}{cc}
\hline DOMÍNIOS QASCI (no de itens, intervalo possível) & Intervalo obtido \\
\hline QASCI escore total (32 itens, 32 a 160) & $72-130$ \\
Sobrecarga emocional (4 itens, 4 a 20) & $4-16$ \\
Implicações na vida pessoal ( 11 itens, 11 a 55) & $11-52$ \\
Sobrecarga financeira (2 itens, 2 a 10) & $2-10$ \\
Reações às exigências ( 5 itens, 5 a 25) & $5-20$ \\
Apoio familiar (2 itens, 2 a 10) & $5-15$ \\
Percepção dos mecanismos de eficácia e controle (3 itens, 3 a 15) & $2-10$ \\
Satisfação com o papel e com o familiar (5 itens, 5 a 25) & $14-25$ \\
\hline
\end{tabular}

Apesar de o estudo informar que os participantes da pesquisa consideram ter conhecimentos e experiência para cuidar dos seus respectivos familiares em 54,28\% das respostas, os cuidadores informais não apresentam preparação técnica e, consequentemente, sentem-se, muitas vezes, desapoiados e impotentes ao amparar o indivíduo assistido (LOPES e MONTEIRO, 2015). O Caderno de Atenção Domiciliar Volume 1 ressalta a importância de o cuidador promover a autonomia e a independência do paciente assistido. O presente estudo demonstrou, no entanto, que $80 \%$ dos participantes da pesquisa sempre sentem que os pacientes assistidos dependem deles.

O Caderno de Atenção Domiciliar Volume 2 salienta a necessidade de suporte da equipe de Atenção Domiciliar aos cuidadores para uma menor sobrecarga e para o desenvolvimento da saúde desses e, até mesmo, dos pacientes. O Caderno de Atenção Domiciliar Volume 1 recomenda, ainda, a formação de grupos de cuidadores para que os mesmos possam compartilhar experiências e saberes, bem como ajudar uns aos outros a reduzir os desgastes pelos quais passam. 
Os estudos realizados por Fernandes e Angelo, Ballarin et al, Aires et al, e Paula et al apresentam resultados semelhantes aos do presente trabalho em relação ao nível de sobrecarga dos cuidadores informais e à piora da saúde dos mesmos pelo exercício da função. Os profissionais de saúde não devem auxiliar apenas aqueles que apresentam dependência, mas também o cuidador informal para que ocorra a prevenção de doenças e a promoção da saúde para ambos os assistidos pelo sistema (LOPES e MONTEIRO, 2015).

A equipe multiprofissional de saúde deve, portanto, além fornecer de suporte ao paciente, atentar-se às necessidades do cuidador, principalmente no ensino ao exercício da função (DE SOUZA et al, 2015; FERNANDES e ANGELO, 2016; BALLARIN et al, 2016; AIRES et al, 2017; DE PAULA et al, 2017), no apoio emocional e no fornecimento de informações visto que o mesmo as procura em fontes informais de informação (FERNANDES e ANGELO, 2016). Esse suporte poderia minimizar a sobrecarga e contribuir para o relacionamento entre o cuidador e o paciente (BALLARIN et al, 2016).

\section{CONSIDERAÇÕES FINAIS}

O estudo permite concluir que há sobrecarga moderada no grupo de cuidadores informais avaliado. Os resultados do estudo devem ser avaliados com cautela visto que a amostra populacional não é significativa. No entanto, o estudo permite uma reflexão acerca da necessidade do reconhecimento das demandas do cuidador informal. É necessário o suporte ao mesmo em prol de uma redução da sobrecarga por meio de uma assistência adequada. O suporte poderia ser realizado por meio do acesso à informação sobre a prática do cuidador informal quando a pessoa passa torna-se um ou, ainda, quando o é. Esse suporte poderia ocorrer no serviço de atenção domiciliar local e nas visitas domiciliares realizadas pela EMAD. Além disso, a assistência deve abranger as necessidades do cuidador informal, sejam emocionais, físicas ou estruturais. Tais sistemas de apoio são importantes para reduzir a sua sobrecarga e aprimorar o exercício da função.

\section{REFERÊNCIAS}

AIRES, M.; MOCELLIN, D.; FENGLER, F. L.; ROSSET, I.; SANTOS, N. O. D.; MACHADO, D. D. O et al. Associação entre responsabilidade filial no cuidado aos pais e sobrecarga dos cuidadores. Revista Brasileira de Enfermagem, v. 70, n. 4, p. 800807, 2017. http://dx.doi.org/10.1590/0034-7167-2017-0133

BALLARIN, M. L. G. S.; BENEDITO, A. C.; KRÖN, C. A.; CHRISTOVAM, D. Sociodemographic profile and burden of informal carevigers of patients assisted in occupational therapy outpatient clinic. Cadernos de Terapia Ocupacional da UFSCar, v. 24, n. 2, 2016. 10.4322/0104-4931.ctoAO0607

BRASIL. Agência Nacional de Vigilância Sanitária. Home care. Disponível em: $<$ http://portal.anvisa.gov.br/resultado-de-

busca? $p \quad p \quad i d=101 \& p \quad p$ lifecycle $=0 \& p \quad p$ state $=$ maximized\& $p$ mode $=v i e w \& p \quad p$ col $i d=$ column$1 \& \mathrm{p} \mathrm{p}$ col count $=1 \& 101$ struts action $=\% 2$ Fasset publisher\%2Fview content\& 101 assetEntryld $=$ 2870410\& 101 type=content\& 101 groupld=219201\& 101 urlTitle=home-

care\&inheritRedirect=true $>$. Acesso em: 6 nov. 2019. 
Agência Nacional de Vigilância Sanitária. Resolução da Diretoria Colegiada - RDC no 11, de 26 de janeiro de 2016. Disponível em: $<$ http://bvsms.saude.gov.br/bvs/saudelegis/anvisa/2006/res0011 2601 2006.html >. Acesso em: 6 nov. 2019.

Ministério da Saúde. Modalidades de Atenção Domiciliar. Disponível em: $<$ http://portalms.saude.gov.br/acoes-e-programas/melhor-em-casa-servico-deatencao-domiciliar/atencao-domiciliar/modalidades-de-atencao-domiciliar>. Acesso em: 6 nov. 2019.

. Ministério da Saúde. Secretaria de Atenção à Saúde. Caderno de Atenção Domiciliar Volume 1. Brasília: Ministério da Saúde; 2012. Disponível em: http://bvsms.saude.gov.br/bvs/publicacoes/caderno atencao domiciliar.pdf

Ministério da Saúde. Secretaria de Atenção à Saúde. Caderno de Atenção Domiciliar Volume 2. Brasília: Ministério da Saúde; 2013. Disponível em: http://bvsms.saude.gov.br/bvs/publicacoes/caderno atencao domiciliar melhor cas a.pdf

CASTRO, L.; DE SOUZA, D. N.; PEREIRA, A.; SANTOS, E.; LOMEO, R.; MENDES, L. et al. Sentimento de culpa e o suporte social no autocuidado das cuidadoras informais familiares. Psicologia: Revista da Associação Portuguesa Psicologia, v. 31, n. 2, 2017. ISSN: 2183-2471.

DE PAULA, S. F.; GEHLEN, M. H.; VENTURA, J.; ZAMBERLAN, C.; RANGEL, R. F.; DE SIQUEIRA, H. C. H. Caregiver's perception about learning for home care. Acta Scientiarum. Health Sciences, v. 39, n. 2, 2017. 10.4025/actascihealthsci.v39i2.33565

DE SOUZA, L. R.; HANUS, J. S.; LIBERA, D.; BOLZAN, L.; SILVA, V. M.; MANGILLI, E. M. et al. Sobrecarga no cuidado, estresse e impacto na qualidade de vida de cuidadores domiciliares assistidos na atenção básica. Cadernos Saúde Coletiva, v. 23, n. 2, 2015. 10.1590/1414-462X201500020063

DO NASCIMENTO, G. G. S. Os cuidados continuados de saúde em Portugal: perspectiva internacional, experiência do paciente na transição e sobrecarga dos cuidadores informais. Portugal, 2015, 367 p. Tese (Doutor em Gestão com Especialização em Recursos Humanos e Comportamento Organizacional, Instituto Universitário de Lisboa.

FERNANDES, C. S.; ANGELO, M. Cuidadores familiares: o que eles necessitam? Uma revisão integrativa. Revista da Escola de Enfermagem da USP, v. 50, n. 4, p. 675-682, 2016. https://doi.org/10.1590/S0080-623420160000500019

GUERRA, H. S.; ALMEIDA, N. A. M.; DE SOUZA, M. R.; MINAMISAVA, R. A. A Sobrecarga do Cuidador Domiciliar. Revista Brasileira em Promoção da Saúde, v. 30, n. 2, p. 179-186, 2017. http://dx.doi.org/10.5020/18061230.2017.p179

LOPES, M. F.; MONTEIRO, M. Cuidadores informais: da vivência à sobrecarga da experiência. Saúde: do Desafio ao Compromisso, p. 39-47, 2015. ISBN: 978-98997708-5-0 
R Core Team. R: A language and environment for statistical computing. R Foundation for Statistical Computing, Vienna, Austria, 2018. Disponível em: https://www.Rproject.org/ 